\title{
Harmonic Analysis in Microgrid and Distributed Energy System Using Harmonic Balance Method
}

\author{
Junwei Lu, \\ School of Engineering, Griffith University, Brisbane, \\ Qld 4111, Australia \\ j.lu@griffith.edu.au \\ Xiaojun Zhao \\ School of Electrical and Electronic Engineering, \\ North China Electric Power University, Baoding, China \\ 158748295@163.com
}

\begin{abstract}
The paper proposes a novel and accurate harmonic analysis method entitled: Harmonic Balance Method (HBM) for Microgrid and distributed energy system (DES). The harmonic balance method can be used to calculate all forms of harmonics generated from nonlinear inductive load, power electronic based inverters and drives in DES, electric machines and saturated transformer. A DC biased High Voltage DC (HVDC) transformer model is used to calculate harmonics current. HBM is an effective harmonic prediction method which can provide accurate harmonic components to the active power filter (APFs).
\end{abstract}

Index Terms--Active power filter, Harmonic Balance method, Harmonic distortion, Harmonic prediction, Renewable energy systems.

\section{INTRODUCTION}

In power systems harmonic distortion is mainly caused by non-linear loads and power electronics used in different devices and distributed renewable energy systems [1]. Harmonics are a distortion of the normal electrical current waveform, generally transmitted by non-linear loads. Power electronics based motor drives, induction machines and saturated transformers, DC/AC inverters used in Microgrid and DES, battery chargers and UPSs are examples of nonlinear loads. Single-phase non-linear loads are prevalent in modern office buildings, while three-phase, non-linear loads are widespread in factories and industrial plants [2], and DC biased power transformers in HVDC power systems and quasi-DC bias problem caused by GMD and GIC in normal power systems [3]. However, the study of these harmonics problems is normally focused on the electrical circuit level, the harmonic problem in the component and structure level (or electromagnetic fields) has not been fully investigated due to a lack of understanding of the characteristics of non-linear electromagnetic fields, and a lack of theory and methodology dealing with harmonics generated from non-linear electromagnetic fields and power electronics.

\author{
Hui Li \\ College of Automation Engineering, Shanghai \\ University of Electric Power, Shanghai, China \\ lihui@shiep.edu.cn
}

In recent years, the presence of non-linear loads and the increasing number of distributed energy resource (DER) units in electrical grids contribute significantly to change the characteristics of voltage and current waveforms in power systems [4]. In this paper we propose an accurate and effective harmonic analysis method that is called: Harmonic Balance Method (HBM) for Microgrid and DES. This method can calculate all forms of harmonics generated from nonlinear inductive loads and saturated transformers; therefore it can provide accurate prediction of harmonic components for active power filters (APFs) and fast control systems. The HBM is a classical technique for studying nonlinear system oscillations in mechanical engineering [5]. The main advantages of this approximate analysis method are that it can be computationally very efficient and that it often gives accurate results along with useful insights into system behavior. In electrical engineering, HBM has been used for nonlinear electromagnetic field analysis [6] in later 1980's. The HBM has been also successfully adopted to analyze the nonlinear electromagnetic filed problems for DC biased HVDC transformer in later 2000's. However, HBM has not been used for analyzing the harmonic problem in electrical power system and power electronics.

This paper intends to provide a detailed concept of HBM and its application in calculating the harmonics caused by non-linear loads in Microgrid and DES. The use of HBM for harmonic prediction in conjunction with the time-domain harmonic detection method (TD-HDM) for nonlinear models is proposed to calculate harmonic current. As a case study of a DC biased HVDC transformer model is presented to prove the concept. The mathematical model of a nonlinear inductive model is derived and used for APF harmonic prediction. Since HBM can give an accurate approximation to the response of the nonlinear system, and further improve the power quality and control performance, the HBM based harmonic prediction method associated with time domain or frequency domain harmonic detection method is recommended to APFs. 


\section{HARMONIC DESTORTION AND REDUCTION}

\section{A. Total Harmonic Distortion (THD) Reduction}

In an attempt to reduce harmonics or THD in Microgrid and DES, and meet the Distribution and Utilization standards [7], various active power filters (APFs) are connected to the Point of Common Coupling (PCC) as illustrated in Fig. 1. Among them the APF integrated with d-Statcom inverter is the most cost effective active power filter, where current sensors mounted upstream or downstream of the APF sense harmonic distortion from the nonlinear load. The current sensors monitor the harmonic distortion on the low voltage AC Bus, and then the APF injects the appropriate correction based on loading at the time to eliminate the distortion. However due to the interference and the delay of signal response, the signal processing time of the control system will be much longer and mismatching sometimes. To provide a synchronized and faster response of harmonic cancellation information, the prediction of harmonic method associated with time domain or frequency domain harmonic detection method will play an important role in APFs.

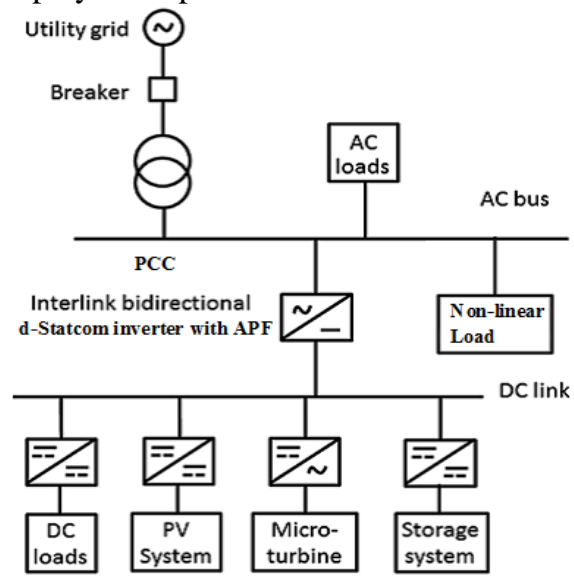

Figure 1. Hybrid AC/DC Microgrid system with d-Statcom and APF

\section{B. Harmonic Detection Methods used in APF}

The harmonic detection method is a common used method in APF which has the capability of determining the harmonics from an input signal such as voltage and current and convert to a numerical model by using a special mathematical algorithm. Then, with the achieved information, the inner (current/voltage) controller is imposed to compensate for the existing harmonic distortion [8]. Different harmonic detection methods used in APFs have their advantages and disadvantages depending on the mathematical algorithms involved, the detection accuracy, the speed, the filter stability, easy and inexpensive implementation, etc. The classification of the harmonic detection methods can be described as two directions: the time-domain harmonic detection method (TD-HDM) and the frequency-domain harmonic detection method (FD-HDM) [8].

The frequency-domain methods are mainly identified with Fourier analysis, rearranged to provide the result as fast as possible with a reduced number of calculations, to allow a real-time implementation in DSPs. The discrete Fourier transform (DFT) is a mathematical transformation for discrete signals, which gives both the amplitude and phase information of the selected harmonic. While the time-domain methods offer increased speed and fewer calculations compared to the frequency-domain methods. Once the harmonics are detected and isolated in the frequency domain, it is just a matter of reconstruction back in the time domain of the reference signal for the inner controller.

\section{HARMONIC ANALYSIS USING HBM}

The HBM can be applied to nonlinear Electromagnetic (EM) field analysis that consists of the harmonics which also satisfy Maxwell's equations. The harmonics generated in EM fields are originated by the three main categories as follows:

- Linear EM device can exhibit harmonics when it is energized by the source that contain harmonics,

- Nonlinear EM device can exhibit harmonics when it is energized by a sinusoidal signal,

- Both linear and nonlinear EM devices can exhibit complex harmonic field by the sources that contain harmonics.

\section{A. Harmonics Generated from Nonlinear Inductive Load}

The current drawn by non-linear loads passes through all of the impedance between the system source and load. This current produces harmonic voltages for each harmonic as it flows through the system impedance. The sum of these harmonic voltages produces a distorted voltage when combined with the fundamental. The voltage distortion magnitude is dependent on the source impedance and the harmonic voltages produced. Figure 2 illustrates how the distorted voltage is created. As illustrated, non-linear loads are typically modeled as a source of harmonic current.

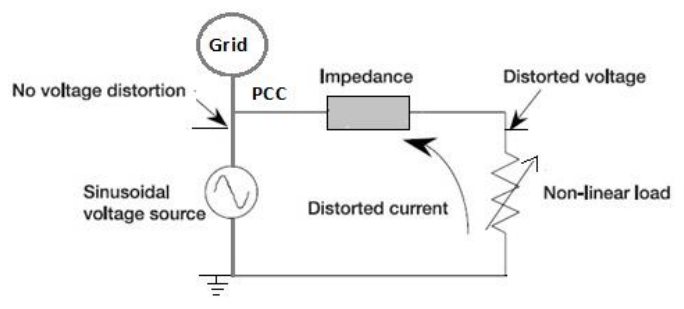

Fig. 2 Harmonic current generated from nonlinear inductive load

Harmonics can be generated from other two major nonlinear components in power system that are rotating machine and saturated transformer models. Rotating machines are considered sources of harmonics because the windings are embedded in slots which are not perfectly sinusoidally distributed resulting in a distorted mmf.

\section{B. HBM used for Nonlinear EM Field}

Nonlinear phenomena in EM fields are normally caused by the nonlinear materials used in various electric machines. The nonlinear materials are normally functions of field strength of EM field which can cause harmonics. Thus, when the timebased quasi-static EM field is applied to the nonlinear material, the electromagnetic characteristics of the material 
will be functions of the EM field that is also time dependent. On the other hand, harmonics can also be generated by power electronic devices and drives, which are largely used in power systems, DER units and micro grids. These power electronic devices and drives are used for power rectifications, power conversions (e.g. dc/dc converter) and inversions (e.g. dc/ac inverter). Both the nonlinear materials and power electronic devices cause harmonic problems in EM fields.

Fig. 3 illustrates the B-H curve of magnetic core. Fig. 3(a) shows the general expression of the B-H curve followed by Fig. 3(b) that presents hysteresis and dc biased cases.

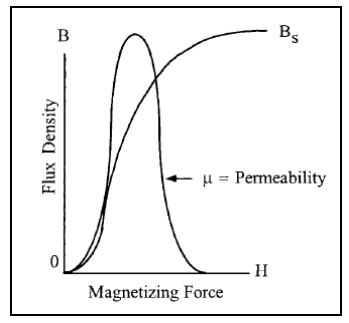

(a)

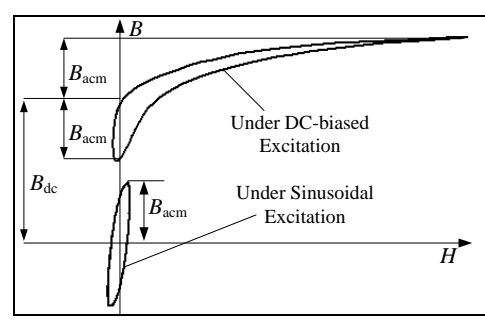

(b)
Fig. 3. B-H curve of magnetic core a) B-H curve of magnetic core in general expression, b) with hysteresis and dc biased case.

\section{1) HBM-Finite Element Method for Nonlinear EM Field}

Based on the Maxwell equations, the expression of harmonics in quasi- static magnetic field can be presented as:

$$
\nabla \times v \nabla \times A+\sigma(\partial A / \partial t+\nabla \varphi)-J_{s}=0
$$

where $\boldsymbol{A}$ is the magnetic vector potential, $\boldsymbol{\varphi}$ is the scalar potential, $\sigma$ is the electrical conductivity, and $\boldsymbol{J}_{\mathrm{s}}$ is the source current density, expressed as:

$$
\begin{aligned}
& A^{i}=A_{0}^{i}+\sum_{k=1}^{\infty}\left\{A_{k s}^{i} \sin (k \omega t)+A_{k c}^{i} \cos (k \omega t)\right\} \\
& \varphi^{i}=\varphi_{0}^{i}+\sum_{k=1}^{\infty}\left\{\varphi_{k s}^{i} \sin (k \omega t)+\varphi_{k c}^{i} \cos (k \omega t)\right\} \\
& J_{s}=J_{0}+\sum_{k=1}^{\infty}\left\{J_{k s} \sin (k \omega t)+J_{k c} \cos (k \omega t)\right\}
\end{aligned}
$$

where the vectors $\boldsymbol{A}_{0}, \boldsymbol{J}_{0}$ and scalar $\varphi_{0}$ are the dc components respectively, and $k_{s}$ and $k_{c}$ represent the $\sin$ and $c o s$ components. In practical applications, as the harmonic $k$ is not infinite, only a finite number for $k$ is required.

The magnetic reluctivity $\boldsymbol{v}$ corresponding to $\boldsymbol{B}(\mathrm{t})$ based on Fig. 3 can be expressed as:

$$
v(t)=H(B(t)) / B(t)=v_{0}+\sum_{k=2 n-2}^{\infty}\left\{v_{k s} \sin (k \omega t)+v_{k c} \cos (k \omega t)\right\}
$$

The Harmonic Balance-Finite Element Method (HBFEM) can approximate the time periodic solution (magnetic potentials, currents, voltages, etc.) with a truncated Fourier series. Besides the frequency components of the excitation (e.g. applied voltages or current), the solution contains harmonics due to nonlinearity (magnetic saturation and nonlinear lumped electrical components), and power electronic devices and drives [9].
For a multi-frequency domain or harmonic domain, based on FEM approach, the governing equation in a 2D case can be rewritten as:

$G=\iint_{S}\left\{\frac{\partial N_{i}}{\partial x} v \frac{\partial A_{k}}{\partial x}+\frac{\partial N_{i}}{\partial y} v \frac{\partial A_{k}}{\partial y}\right\} d x d y-\iint_{S}\left\{J_{0}-\sigma \frac{\partial A_{k}}{\partial t}\right\} N_{i} d x d y=0$

where magnetic vector potential $\boldsymbol{A}_{\mathrm{k}}$ and current density $\boldsymbol{J}_{0}$ consist of $k$ th harmonics and dc component are expressed in Equations (2) and (4).

Substituting equations (2) and (4) to (6), the single element matrix equation of HBFEM can be derived as follows:

$$
\begin{aligned}
& G^{e}=\frac{1}{4 \Delta^{e}}\left[\begin{array}{ccc}
\left(b_{1} b_{1}+c_{1} c_{1}\right) D & \left(b_{1} b_{2}+c_{1} c_{2}\right) D & \left(b_{1} b_{3}+c_{1} c_{3}\right) D \\
\left(b_{2} b_{1}+c_{2} c_{1}\right) D & \left(b_{2} b_{2}+c_{2} c_{2}\right) D & \left(b_{2} b_{3}+c_{2} c_{3}\right) D \\
\left(b_{3} b_{1}+c_{3} c_{1}\right) D & \left(b_{3} b_{2}+c_{3} c_{2}\right) D & \left(b_{3} b_{3}+c_{3} c_{3}\right) D
\end{array}\right]\left\{\begin{array}{l}
A_{1}^{e} \\
A_{2}^{e} \\
A_{3}^{e}
\end{array}\right\} \\
& +\frac{\sigma \omega \Delta^{e}}{12}\left[\begin{array}{ccc}
2 N & N & N \\
N & 2 N & N \\
N & N & 2 N
\end{array}\right]\left\{\begin{array}{l}
A_{1}^{e} \\
A_{2}^{e} \\
A_{3}^{e}
\end{array}\right\}-\left\{\begin{array}{l}
K_{1}^{e} \\
K_{2}^{e} \\
K_{3}^{e}
\end{array}\right\}=\left[S^{e}\right]\left\{A_{i}^{e}\right\}+\left[M^{e}\right]\left\{A_{i}^{e}\right\}-\left[K_{i}^{e}\right]
\end{aligned}
$$

where D is The Reluctivity Matrix and the magnetic of its reluctivity coefficients are determined by the Fourier coefficients in Equation (5).

The magnetic reluctivity coefficient matrix, $D$ can be derived as Equation (8), and the matrix $N$ that is called the time Harmonic Matrix is a constant concerned with harmonic orders can be expressed by Equation (9). The harmonic matrix $N$ can include dc components, as well as even and order harmonics depending on the application problems in the quasi-steady-state magnetic field.

$$
\begin{aligned}
& D=\frac{1}{2}\left[\begin{array}{cccccccc}
2 v_{0} & v_{1 s} & v_{1 c} & v_{2 s} & v_{2 c} & v_{3 s} & v_{3 c} & \cdots \\
2 v_{1 s} & 2 v_{0}-v_{2 c} & v_{2 s} & v_{1 c}-v_{3 c} & -v_{1 s}+v_{3 s} & v_{2 c}-v_{4 c} & -v_{2 s}+v_{4 s} & \cdots \\
2 v_{1 c} & & 2 v_{0}+v_{2 c} & v_{1 s}+v_{3 s} & v_{1 c}+v_{3 c} & v_{2 s}+v_{4 s} & v_{2 c}+v_{4 c} & \cdots \\
2 v_{2 s} & & & 2 v_{0}-v_{4 c} & v_{4 s} & v_{1 c}-v_{5 c} & -v_{1 s}+v_{5 s} & \cdots \\
2 v_{2 c} & & & & 2 v_{0}+v_{4 c} & v_{2 s}+v_{4 s} & v_{1 c}+v_{5 c} & \cdots \\
2 v_{3 s} & & \text { Symmetry } & & & 2 v_{0}-v_{6 c} & v_{6 s} & \cdots \\
2 v_{3 c} & & & & & & 2 v_{0}+v_{6 c} & \cdots \\
\vdots & \vdots & \vdots & \vdots & \vdots & \vdots & \vdots & \ddots
\end{array}\right] \\
& N=\left[\begin{array}{cccccccc}
0 & 0 & 0 & 0 & 0 & 0 & 0 & \cdots \\
0 & 0 & -1 & 0 & 0 & 0 & 0 & \cdots \\
0 & 1 & 0 & 0 & 0 & 0 & 0 & \cdots \\
0 & 0 & 0 & 0 & -2 & 0 & 0 & \cdots \\
0 & 0 & 0 & 2 & 0 & 0 & 0 & \cdots \\
0 & 0 & 0 & 0 & 0 & 0 & -3 & \cdots \\
0 & 0 & 0 & 0 & 0 & 3 & 0 & \cdots \\
\vdots & \vdots & \vdots & \vdots & \vdots & \vdots & \vdots & \ddots
\end{array}\right]
\end{aligned}
$$

The $[\mathrm{M}]$ matrix including the time harmonic matrix $N$ can be expressed as:

$$
[M]=\frac{\omega \sigma \Delta^{e}}{12}\left[\begin{array}{ccc}
2 N & N & N \\
N & 2 N & N \\
N & N & 2 N
\end{array}\right]
$$

If only the fundamental frequency and third harmonics are considered, $\{\mathbf{A}\}$ and $\{\mathbf{K}\}$ can be expressed as: 
$\left\{A_{i}^{e}\right\}=\left(\left\{A_{k}^{i}\right\}^{T} \quad\left\{A_{k}^{i}\right\}^{T} \quad\left\{A_{k}^{i}\right\}^{T}\right)^{\prime}=$

$\left\{\begin{array}{llllllllllll}A_{1 s}^{1} & A_{1 c}^{1} & A_{3 s}^{1} & A_{3 c}^{1}, & A_{1 s}^{2} & A_{1 c}^{2} & A_{3 s}^{2} & A_{3 c}^{2}, & A_{1 s}^{3} & A_{1 c}^{3} & A_{3 s}^{3} & A_{3 c}^{3}\end{array}\right\}^{T}$

$\left\{K_{i}^{e}\right\}=\left(\left\{K_{1}\right\}^{T} \quad\left\{K_{2}\right\}^{T} \quad\left\{K_{3}\right\}^{T}\right)=\frac{\Delta^{e}}{3}\left\{\begin{array}{llllll}J_{1 s} & J_{1 c} & J_{3 s} & J_{3 c}, & \cdots & \cdots\end{array}\right\}^{T}$

Finally, the generalized HBFEM system matrix can be obtained as:

$[S]\left\{A_{k}\right\}+[M]\left\{A_{k}\right\}-\left[G_{k}\right]\left\{J_{k}\right\}=([S]+[M])\left\{A_{k}\right\}-[K]==0$

\section{HBM for Nonlinear Magnetic Circuit}

\section{1) HBM for Magnetic Circuit}

The current drawn by non-linear loads passes through all of the impedance between the system source and load. This current produces harmonic voltages as it flows through the system impedance. The sum of these voltage harmonics causes distortions on the voltage waveform. Fig. 2 illustrates the equivalent circuit of a typical electrical network with inductive load. As mentioned, the distorted voltage is occurred at the load side due to the distorted current flowing through the network impedances. Therefore, non-linear loads are typically modeled as a source of harmonic current. The HBM technique would be considered as a reliable solution to reduce the generated harmonics in these networks.

Input voltage $\mathrm{V}_{\mathrm{k}}$ can be pure sinewave from inverter or harmonic voltage from poor inverter:

$V_{k, i n}=\sum_{k=n}^{\infty}\left\{V_{k s, i n} \sin (k \omega t)+V_{k c, i n} \cos (k \omega t)\right\}$

The load current is expressed as:

$I_{k}=\sum_{k=n}^{\infty}\left\{I_{k s} \sin (k \omega t)+I_{k c} \cos (k \omega t)\right\}$

From the magnetic circuit of a nonlinear load:

$$
H \ell=N i_{k}=\mathfrak{R}_{k} \Phi_{k}
$$

where $\mathrm{H}$ is magnetic field intensity, $\ell$ is the mean length of the magnetic core, $\mathrm{N}$ is the winding turn number, $\mathfrak{R}$ is $\mathrm{B}-\mathrm{H}$ curve with hysteresis characteristics and dc biased condition, as shown in Fig. 3, the magnetic reluctance, $\Phi$ is magnetic flux expressed as follow:

$\Phi_{k}=\sum_{k=n}^{\infty}\left\{\Phi_{k s} \sin (k \omega t)+\Phi_{k c} \cos (k \omega t)\right\}$

The magnetic reluctance of $\mathfrak{R}$ can be obtained from the magnetic reluctivity $v$ in Eq. (5), and B-H curve and magnetic circuit theory, as shown in Eq. (18)

$$
\begin{aligned}
& \mathfrak{R}_{k}=\sum_{k=n}^{\infty}\left\{\mathfrak{R}_{k s} \sin (k \omega t)+\mathfrak{R}_{k c} \cos (k \omega t)\right\} \\
& =\frac{\ell}{A} \frac{H(B(t))}{B(t)}=\frac{\ell}{A} \sum_{k=n}^{\infty}\left\{v_{k s} \sin (k \omega t)+v_{k c} \cos (k \omega t)\right\}
\end{aligned}
$$

where the $l$ and $\mathbf{A}$ are the average mean length of magnetic circuit and cross area of magnetic circuit respectively.

Applying Kirchhoff's Voltage Law for the nonlinear network in Fig. 2, the relationship between the input voltage and network impedance and nonlinear load can be obtained as bellow:

$V_{i n}=Z_{L k} i_{k}+N \frac{d \Phi_{k}}{d t}=Z_{L k} i_{k}+N k \omega \Phi_{k}$

where $\boldsymbol{N}$ is the winding number of inductive load and $\boldsymbol{k}$ is harmonic number. $Z_{\mathrm{LK}}$ is the impedance including transmission line $\mathrm{R}_{0}$ and $\mathrm{L}_{\mathrm{T}}$, and load winding resistance, $\mathrm{R}_{\mathrm{L}}$

$\left[Z_{L k}\right]=\left[\begin{array}{cc}\left(R_{0}+R_{L}\right) & -k \omega L_{T} \\ \left.k \omega L_{T}\right) & \left(R_{0}+R_{L}\right)\end{array}\right]=\left[\begin{array}{cccc}Z_{0 k} & 0 & 0 & \cdots \\ 0 & Z_{1 k} & 0 & \cdots \\ 0 & 0 & Z_{2 k} & \ldots \\ \vdots & \vdots & \vdots & \ddots\end{array}\right]$

Combining the magnetic equivalent circuit (16) and electric circuit equations (19), when each coefficient at sine- and cosine-components is equated, the system matrix equation of nonlinear inductive model can be obtained as:

$\left\{\begin{array}{c}0 \\ V_{k, \text { in }}\end{array}\right\}=\left\{\begin{array}{c}\Phi_{k} \\ I_{k}\end{array}\right\}\left[\begin{array}{cc}N & \mathfrak{R}_{k} \\ Z_{L k} & N k \omega\end{array}\right]$

The matrix $\boldsymbol{k}$ is a constant concerned with harmonic orders and is called the HARMONIC MATRIX. The matrix of harmonic number $\boldsymbol{k}$ for inductive load:

$k=\left[\begin{array}{ccccccc}0 & -1 & 0 & 0 & 0 & 0 & \cdots \\ 1 & 0 & 0 & 0 & 0 & 0 & \cdots \\ 0 & 0 & 0 & -3 & 0 & 0 & \cdots \\ 0 & 0 & 3 & 0 & 0 & 0 & \ldots \\ 0 & 0 & 0 & 0 & 0 & -5 & \cdots \\ 0 & 0 & 0 & 0 & 5 & 0 & \ldots \\ \cdots & \cdots & \cdots & \cdots & \ldots & \ldots & \ddots\end{array}\right]$

The reluctance matrix can be obtained as bellow:

$$
\Re_{k}=\frac{1}{2}\left[\begin{array}{ccccc}
2 \mathfrak{R}_{0}-\mathfrak{R}_{2 c} & \mathfrak{R}_{2 s} & \mathfrak{R}_{2 c}-\mathfrak{R}_{4 c} & -\mathfrak{R}_{2 s}+\mathfrak{R}_{4 s} & \ldots \\
& 2 \mathfrak{R}_{0}+\mathfrak{R}_{2 c} & \mathfrak{R}_{2 s}+\mathfrak{R}_{4 s} & \mathfrak{R}_{2 c}+\mathfrak{R}_{4 c} & \\
& & 2 \Re_{0}-\mathfrak{R}_{6 c} & \mathfrak{R}_{6 s} & \\
& \text { Symmetry } & & 2 \mathfrak{R}_{0}+\mathfrak{R}_{6 c} & \\
\vdots & & & & \ddots
\end{array}\right]
$$

After solving the matrix equation (21), all unknown harmonic components can be obtained as following:

$I_{k}=\left\{I_{1 s}, I_{1 c}, I_{3 s}, I_{3 c}, \cdots I_{k s}, I_{k c}\right\}^{T}$

$\Phi_{k}=\left\{\Phi_{1 s}, \Phi_{1 c}, \Phi_{3 s}, \Phi_{3 c}, \cdots \Phi_{k s}, \Phi_{k c}\right\}^{T}$

The distorted voltage at the load according to Faraday's law can be obtained from matrix equation (21) as:

$V_{k, \text { Load }}=N \frac{d \Phi_{k}}{d t}=\Phi_{k} N k \omega$

$=\sum_{k=n}^{\infty}\left\{V_{k s, \text { Load }} \sin (k \omega t)+V_{k c, \text { Load }} \cos (k \omega t)\right\}$ 
The harmonic current can be also obtained from matrix equation (21),

$I_{k}=\sum_{k=n}^{\infty}\left\{I_{k s} \sin (k \omega t)+I_{k c} \cos (k \omega t)\right\}$

\section{APPLICATIONS OF HBM IN POWER SYSTEM AND APF}

\section{A. HBFEM for DC Biased Transformer Analysis}

Transformer saturation is not a major issue with renewable generation sources. However, the inverter technology used in one instance resulted in injecting small levels of DC current into the transformer and the resulting transformer saturation created significant harmonic currents. In the wake of this event, a study was done to evaluate transformer saturation. The results of the study show that several amperes of DC current injection on the low side could easily cause transformer core saturation and result in very high levels of even harmonics (2nd and 4th) on the high side. If the DC current injection is reduced, the harmonic amplification is reduced. Once the DC current infection was reduced to $25 \%$ of the measured values, there was no longer any saturation causing amplification of harmonics on the high side [9].

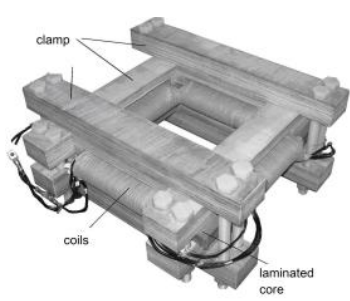

(a)

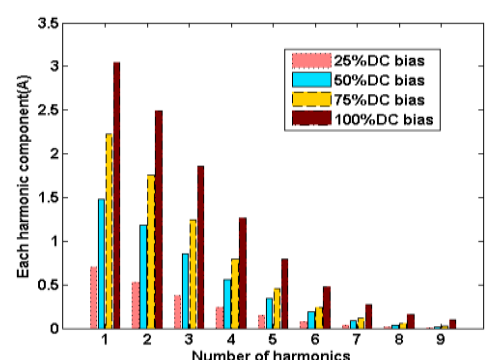

(b)
Fig. 4 (a) DC Biased transformer using Epstein frame-like core model, (b) Each harmonic component of exciting current under different DC bias $\left(U_{\mathrm{m}}=\right.$ $U_{\mathrm{m}, 3}=240 \mathrm{~V} ; B_{\mathrm{m}}=B_{\mathrm{m}, 3}=0.88 \mathrm{~T}$ ) [9].

\section{B. HBM used for Harmonic Prediction in APF}

To apply HBM to APFs used in Microgrid and DES as shown in Fig. 1, a d-statcom inverter with built-in APF is required for implementing this technique, where a simplified nonlinear electrical network as illustrated in Fig. 2 is used to implement the HBM. TD-HDM can be worked with HBM directly in APF where the control system of the d-statcom inverter with a built-in APF is able to predict the harmonic current and voltage cross the load terminal in the network. Therefore the harmonic cancellation process can be effectivelly and accuratly carried out with HBM and TD$\mathrm{HDM}$ as illustrated in Fig. 5.

As shown in Fig. 5, the HBM and TD-HDM based APF model has three main inputs comprised of the load current, $I_{\mathrm{k}}$, voltages at the point of common coupling (PCC), $V_{\mathrm{k}}$ and at load terminal $V_{\mathrm{L}}$. The load premeters, $N, R_{\mathrm{k}}$, and $l$ will be installed in the HBM controller. Using Equations (26) and (27), the harmonics of current and voltage at load terminal can be predicted and extracted accordingly, the accurate reference signal from $\mathrm{HBM}$ without any delay can be generated and compared with real time singnle obtaiend from TD-HDM for control system. As this operation is performed by mathematical calculation of all harmonics associated with the detected current $I_{\mathrm{k}}$ and $V_{\mathrm{k}}$ data to controller, the system operates without any noise or any interference and with the highest speed compared with the conventional methods used for APFs.

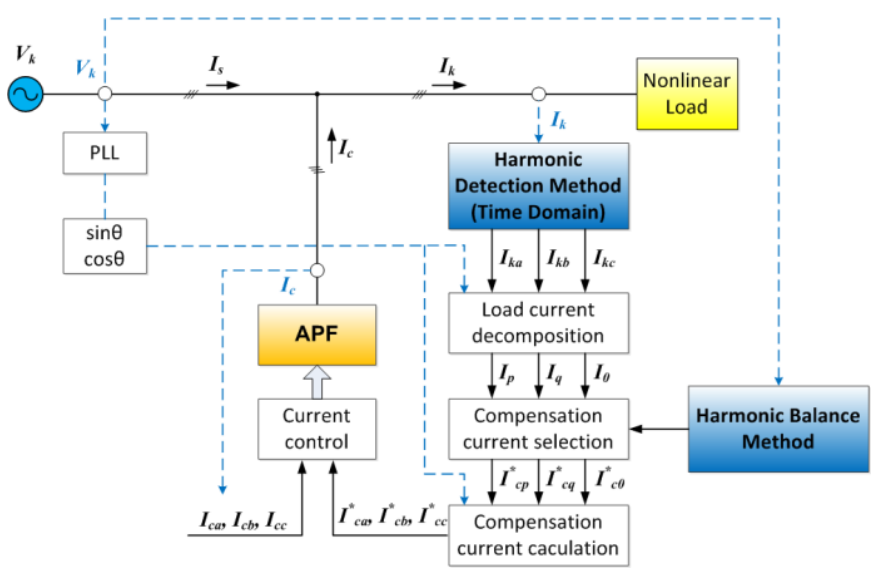

Fig. 5 APF using Harmonic Prediction and Harmonic Detection Method and Control algorithm

\section{ACKNOWLEDGMENT}

This work is supported by Shanghai International Science \& Technology Cooperation Program (No. 15220710500).

\section{REFERENCES}

[1] Larry Ray, P.E. Louis Hapeshis, P.E. "Power System Harmonic Fundamental Considerations: Tips and Tools for Reducing Harmonic Distortion in Electronic Drive Applications," Schneider Electric, October 2011/AT313.

[2] Ioannis Bouloumpasis, Panagis Vovos, Konstantinos Georgakas and Nicholas A. Vovos, Current Harmonics Compensation in Microgrids Exploiting the Power Electronics Interfaces of Renewable Energy Sources, Energies 2015, 8, 2295-2311.

[3] IEEE Power and Energy Society Technical Council Task Force on Geomagnetic Disturbances, "Geomagnetic Disturbances," IEEE Power \& Energy Magazine, July/August 2013, pp 71-78.

[4] IEEE Guide for Applying Harmonic Limits on Power Systems Unpublished Draft, IEEE Std P519.1TM/D9a, January, 2004.

[5] A. Tesi, E. H. Abed, R. Genesio, and H. O. Wang, "Harmonic balance analysis of period-doubling bifurcations with implications for control of nonlinear dynamics," Automatica, vol. 32, pp. 1255-1271, 1996.

[6] J. Lu, X. Zhao and Y. Sotoshi, Harmonic Balance Finite Element Method and Its Application in Nonlinear Electromagnetics and Power System, in press by Wiley, June 2016.

[7] IEEE Std. 519-2014 (Revision of IEEE Std. 519,1992), "IEEE Recommended Practices and Requirements for Harmonic Control in Electric Power Systems", Approved 27 March 2014.

[8] L. Asiminoaei, F. Blaabjerg, and S. Hansen, "Detection is keyHarmonic detection methods for active power filter applications," Industry Applications Magazine, IEEE, vol. 13, pp. 22-33, 2007.

[9] J. Lu, X. Zhao and Y. Sotoshi, Harmonic Balance Finite Element Method and Its Application in Nonlinear Electromagnetics and Power System, in press by Wiley, June 2016. 